\title{
Explicit Solutions of the Extended Skorokhod Problems in Affine Transformations of Time-Dependent Strata
}

\author{
Marek Slaby \\ Department of Mathematics and Computer Science, Fairleigh Dickinson University, 285 Madison Avenue, M-ZN2-02, Madison, \\ NJ 07940, USA
}

Correspondence should be addressed to Marek Slaby; mslaby@fdu.edu

Received 11 March 2021; Accepted 5 June 2021; Published 2 July 2021

Academic Editor: Marek T. Malinowski

Copyright (C) 2021 Marek Slaby. This is an open access article distributed under the Creative Commons Attribution License, which permits unrestricted use, distribution, and reproduction in any medium, provided the original work is properly cited.

The goal of this paper is to expand the explicit formula for the solutions of the Extended Skorokhod Problem developed earlier for a special class of constraining domains in $\mathbb{R}^{n}$ with orthogonal reflection fields. We examine how affine transformations convert solutions of the Extended Skorokhod Problem into solutions of the new problem for the transformed constraining system. We obtain an explicit formula for the solutions of the Extended Skorokhod Problem for any $\mathbb{R}^{n}$ - valued càdlàg function with the constraining set that changes in time and the reflection field naturally defined by any basis. The evolving constraining set is a region sandwiched between two graphs in the coordinate system generating the reflection field. We discuss the Lipschitz properties of the extended Skorokhod map and derive Lipschitz constants in special cases of constraining sets of this type.

\section{Introduction}

The Skorokhod Problem (SP) has its origin in [1], Skorokhod's 1961 paper Stochastic equations for diffusions in a bounded region. It was extended by Tanaka in [2] to simple multidimensional cases and to more general cases by Harrison and Reiman in [3] and by Lions and Sznitman in [4].

Since then, it has been studied extensively in many directions. The Skorokhod map (SM), a part of its solution, together with its slightly more general version, the extended Skorokhod map (ESP), constitute a very important tool in studying processes whose values are restricted to some domain by a constraining force acting along directions specified for each point on the boundary. They found the most significant applications in studying reflected diffusion processes and queueing networks. Two of the most recent directions are particularly relevant to this work: the search for an explicit formula for the solutions and the introduction of time-dependent barriers. In the one-dimensional case, the first explicit formulas were developed in [5] for the solutions of the SP and in [6] for the solutions of the ESP. In addition, the results in [6] allowed also for the constraining interval to change in time. Somewhat different explicit formulas were developed independently by the author in [7] for the solutions of the SP and in [8] for ESP. In both papers, timedependent boundaries were considered.

The $\mathbb{R}^{n}$-valued case however is obviously much more complicated. In [9], the author managed to derive an explicit formula for solutions of the ESP for a special class of timedependent constraining domains called strata with orthogonal reflection fields. It is those results that we intend to generalize now onto a much larger class of constraining domains and more general reflection fields.

In [9], we have introduced a special kind of a constraining set called a stratum. We now introduce its generalization. Let $E=\left\{\mathbf{e}_{1}, \mathbf{e}_{2}, \ldots, \mathbf{e}_{n}\right\}$ be the standard orthonormal basis and $V=\left\{\mathbf{v}_{1}, \mathbf{v}_{2}, \ldots, \mathbf{v}_{n}\right\}$ be any basis of $\mathbb{R}^{n}$. We will use $\left(x^{1}, x^{2}, \ldots, x^{n}\right)_{V}$ to represent the vector $\mathbf{x}=$ $x^{1} \mathbf{v}_{1}+x^{2} \mathbf{v}_{2}+\cdots+x^{n} \mathbf{v}_{n}$ in terms of its coordinates with respect to $V$. The subscript will be omitted when $V$ is the standard orthonormal basis.

Definition 1. A closed set $G$ in $\mathbb{R}^{n}$ will be called a quasistratum if there is a basis $V$ such that 


$$
G=\left\{\left(x^{1}, x^{2}, \ldots, x^{n}\right)_{V}: x^{i} \in\left[a^{i}, b^{i}\right] \text { for } i=1,2, \ldots, n-1, x^{n} \in\left[A\left(x^{1}, \ldots, x^{n-1}\right), B\left(x^{1}, \ldots, x^{n-1}\right)\right]\right\}
$$

where $a^{i} \leq b^{i}$ for $i=1,2, \ldots, n-1$ and $A, B$ are two realvalued continuous functions defined on $\left[a^{1}, b^{1}\right]$ $\times \cdots \times\left[a^{n-1}, b^{n-1}\right]$ such that $A(\mathbf{x}) \leq B(\mathbf{x})$ for every $\mathbf{x}$. For short, we will write

$$
G=S^{V}\left(\left[a^{1}, b^{1}\right] \times \cdots \times\left[a^{n-1}, b^{n-1}\right],[A, B]\right) .
$$

The superscript will be omitted when $V$ is a standard orthonormal basis. In the special case when $A$ and $B$ are constant functions, $G$ will be called a quasiblock.

In two dimensions, a quasiblock is simply a parallelogram, and in three dimensions, it is a parallelepiped. In general, a quasiblock in $\mathbb{R}^{n}$ is a parallelotope. This perhaps not quite popular name was introduced by H.S.M. Coxeter in [10]. Alternatively, a quasiblock in $\mathbb{R}^{n}$ can be described as an $n$-dimensional parallelepiped.

Note that, in the special case, when $V$ is an orthonormal basis, the quasistratum becomes a stratum and a quasiblock becomes a block in the sense of Definition 2.1 in [9]. By $T_{V}$, we will denote the unique linear transformation $T_{V}: \mathbb{R}^{n} \longrightarrow \mathbb{R}^{n}$ mapping the standard orthonormal basis onto $V$, such that $T_{V}\left(\mathbf{e}_{i}\right)=\mathbf{v}_{i}$ for $i=1,2, \ldots, n$. Then,

$$
S^{V}\left(\left[a^{1}, b^{1}\right] \times \cdots \times\left[a^{n-1}, b^{n-1}\right],[A, B]\right)=T_{V}(G),
$$

where $G=S\left(\left[a^{1}, b^{1}\right] \times \cdots \times\left[a^{n-1}, b^{n-1}\right],[A, B]\right)$. Note that $T_{V}$ can be represented by a matrix whose columns are $\mathbf{v}_{1}, \mathbf{v}_{2}, \ldots, \mathbf{v}_{n}$. Any invertible affine transformation of $\mathbb{R}^{n}$ can be represented as a composition of a translation with $T_{V}$ for some basis $V$.

Definition 2. A family of pairs $\left\{\left(G_{t}, d_{t}(\cdot)\right): t \geq 0\right\}$ will be called an evolving quasistratum constraining system if there is a basis $V$ such that $G_{t}=S^{V}\left(\left[\alpha_{t}^{1}, \beta_{t}^{1}\right] \times \cdots \times\right.$ $\left.\left[\alpha_{t}^{n-1}, \beta_{t}^{n-1}\right],\left[A_{t}, B_{t}\right]\right),\left\{G_{t}: t \geq 0\right\}$ is càdlàg with respect to the Hausdorff distance between constraining sets, and $d_{t}$ satisfies the following conditions.

For any $\mathbf{x}=\left(x^{1}, x^{2}, \ldots, x^{n}\right)_{V}$ on the boundary of $G_{t}$,

$$
d_{t}(\mathbf{x})=d_{t}^{V}(\mathbf{x})=\left\{\sum_{i \in I_{t}^{V}} r^{i} \mathbf{v}_{i}-\sum_{i \in J_{t}^{V}} r^{i} \mathbf{v}_{i}: r^{i} \geq 0, \text { for } i \in I_{t}^{V}(\mathbf{x}) \cup J_{t}^{V}(\mathbf{x})\right\} \text {, }
$$

where

$$
\begin{aligned}
I_{t}^{V}(\mathbf{x}) & =\left\{i: 1 \leq i<n \text { and } x^{i}=\alpha_{t}^{i} \text { or } i=n \text { and } x^{n}=A\left(x^{1}, x^{2}, \ldots, x^{n-1}\right)\right\} \\
J_{t}^{V}(\mathbf{x}) & =\left\{i: 1 \leq i<n \text { and } x^{i}=\beta_{t}^{i} \text { or } i=n \text { and } x^{n}=B\left(x^{1}, x^{2}, \ldots, x^{n-1}\right)\right\}
\end{aligned}
$$

Finally, $d_{t}(\mathbf{x})=0$ for any $\mathbf{x}$ in the interior of $G_{t}$.

In the special case when $G_{t}$ is a quasiblock for every $t \geq 0$, the evolving quasistratum constraining system will be called an evolving quasiblock constraining system.

Throughout this paper, $D\left([0, \infty), \mathbb{R}^{n}\right)$ will denote the space of $\mathbb{R}^{n}$-valued right continuous functions with left limits, the so-called càdlàg functions, defined on $[0, \infty)$. The subspace of $D\left([0, \infty), \mathbb{R}^{n}\right)$ consisting of functions taking values in $G$ at $t=0$ will be denoted by $D_{G}\left([0, \infty), \mathbb{R}^{n}\right)$.

Remark 1. Let $\left\{\left(G_{t}, d_{t}(\cdot)\right): t \geq 0\right\}$ be an orthogonal evolving stratum constraining system with $G_{t}=S$ $\left(\left[\alpha_{t}^{1}, \beta_{t}^{1}\right] \times \cdots \times\left[\alpha_{t}^{n-1}, \beta_{t}^{n-1}\right],\left[A_{t}, B_{t}\right]\right)$, let $V$ be any basis, and let $d_{t}^{V}(\mathbf{x})=T_{V}\left(d_{t}\left(T_{V}^{-1}(\mathbf{x})\right)\right)$. Then, $\left(\left(T_{V} G_{t}, d_{t}^{V}\right): t>0\right)$ is a quasistratum constraining system. In particular,

$$
\begin{aligned}
T_{V} G_{t} & =S^{V}\left(\left[\alpha_{t}^{1}, \beta_{t}^{1}\right] \times \cdots \times\left[\alpha_{t}^{n-1}, \beta_{t}^{n-1}\right],\left[A_{t}, B_{t}\right]\right), \\
d_{t}^{V}(\mathbf{x}) & =\left\{\sum_{i \in I_{t}^{V}} r^{i} \mathbf{v}_{i}-\sum_{i \in J_{t}^{V}} r^{i} \mathbf{v}_{i}: r^{i} \geq 0, \text { for } i \in I_{t}^{V}(\mathbf{x}) \cup J_{t}^{V}(\mathbf{x})\right\} .
\end{aligned}
$$

Proof. Equation (6) follows immediately from (3). Applying (4) to both bases $V$ and $E$ leads to $T_{V} \circ d_{t}(\mathbf{x})=d_{t}^{V} \circ T_{V}(\mathbf{x})$, which implies (7).

Given a time-dependent constraining system $\left\{\left(G_{t}, d_{t}(\cdot)\right): t \geq 0\right\}$ in $\mathbb{R}^{n}$ and an $\mathbb{R}^{n}$-valued càdlàg function $\psi$ such that $\psi(0) \in G_{0}$, the Extended Skorokhod Problem (ESP) is to find a pair of functions $(\phi, \eta)$, both from $D\left([0, \infty), \mathbb{R}^{n}\right)$ such that $\phi=\psi+\eta$, and for every $t \geq 0$, the following conditions are satisfied:

$$
\begin{aligned}
& \phi(t) \in G_{t}, \\
& \eta(t)-\eta(s) \in \overline{\operatorname{co}}\left[\cup_{u \in(s, t]} d_{u}(\phi(u))\right], \quad \text { for every } s \in[0, t]
\end{aligned}
$$

$$
\eta(t)-\eta(t-) \in d_{t}(\phi(t))
$$

where $\eta(t-)$ is the limit from the left of $\eta$ at $t$ and $\overline{\operatorname{co}}(A)$ is the closure of the convex hull of the set $A$. The function $\phi$ is called the extended Skorokhod map (ESM).

The ESP was introduced in [11] and is a generalization of the SP originally introduced in [1] in the real-valued case and further developed in the multidimensional case in [2]. The technical difference is that, in the Skorokhod Problem, the constraining function $\eta$ is required to have finite variation. The discussions in this paper are restricted to the ESP, however, corresponding modified versions of all results could be formulated for the SP as well. The SP in timedependent domains was first studied by Constantini et al. in [12]. Further work in time-dependent intervals was continued by Burdzy et al. in [13], by Burdzy et al. in [6], and by Slominski and Wojciechowski in [14]. More general multidimensional time-dependent domains were studied by Nyström and Önskog in [15].

The explicit formula was first derived in [5] for the solutions of the SP on $[0, a]$. Explicit formulas for the solutions of ESP on the time-dependent interval in $\mathbb{R}$ were 
developed by Burdzy et al. in [6] and independently by the author in [8]. In [9], the author further extended the explicit formulas onto the solutions of ESP on strata in $\mathbb{R}^{n}$ with orthogonal constraining fields.

The SM and the ESM are natural tools used to study reflected deterministic and stochastic processes. In particular, a lot of research was done in the area of reflected diffusions, where the solutions of the SP or ESP are applied. Some of the recent papers include [16-19].

Significant contributions on Lipschitz continuity properties of the SM came from Dupuis and Ishii in [20] and Dupuis and Ramanan in [21, 22].

Very useful in working with ESP are projections onto the constraining domains. In the one-dimensional case, we define

$$
\pi_{a, b}= \begin{cases}a, & \text { if } x \leq a, \\ x, & \text { if } a \leq x \leq b \\ b, & \text { if } x \geq b\end{cases}
$$

We can use it then to define a projection on a quasiblock in $\mathbb{R}^{n}$. If $G=S^{V}\left(\left[a^{1}, b^{1}\right] \times \cdots \times\left[a^{n}, b^{n}\right]\right)$, then $\pi_{G}(\mathbf{x})=\left(\pi_{a^{1}, b^{1}}\left(x^{1}\right), \pi_{a^{2}, b^{2}}\left(x^{2}\right), \ldots, \pi_{a^{n}, b^{n}}\left(x^{n}\right)\right)_{V}$. To define a projection on a quasistratum, we need to extend first functions $A$ and $B$ onto $\mathbb{R}^{n-1}$. An extension of $A$ : $\left[a^{1}, b^{1}\right] \times$ $\cdots \times\left[a^{n-1}, b^{n-1}\right] \longrightarrow \mathbb{R}$ onto $\mathbb{R}^{n-1}$, denoted by $\bar{A}$ will be defined by

$$
\bar{A}_{t}\left(x^{1}, x^{2}, \ldots, x^{n-1}\right)=A_{t}\left(\pi_{\alpha_{t}^{1}, \beta_{t}^{1}}\left(x^{1}\right), \pi_{\alpha_{t}^{2}, \beta_{t}^{2}}\left(x^{2}\right), \ldots, \pi_{\alpha_{t}^{n-1}, \beta_{t}^{n-1}}\left(x^{n-1}\right)\right) .
$$

Similarly, we define $\bar{B}$, the extension of $B$ onto $\mathbb{R}^{n-1}$. Finally, we define the projection onto a quasistratum $G=$ $S^{V}\left(\left[a^{1}, b^{1}\right] \times \cdots \times\left[a^{n-1}, b^{n-1}\right],[A, B]\right)$ by

$$
\pi_{G}(\mathbf{x})=\left(\pi_{a^{1}, b^{1}}\left(x^{1}\right), \ldots, \pi_{a^{n-1}, b^{n-1}}\left(x^{n-1}\right), \pi_{\bar{A}\left(x^{1}, \ldots, x^{n-1}\right), \bar{B}\left(x^{1}, \ldots, x^{n-1}\right)}\left(x^{n}\right)\right)_{V} .
$$

The solution of ESP for $\psi$ is particularly easy to find when $\psi$ is a piecewise constant function with a finite number of jumps.

Example 1. Let $V=\left\{\mathbf{v}_{1}, \mathbf{v}_{2}, \ldots, \mathbf{v}_{n}\right\}$ be any basis of $\mathbb{R}^{n}$, let $0=t_{0}<t_{1}<t_{2}<\cdots<t_{m-1}<t_{m}=\infty$, and let $G_{t}=S^{V}$ $\left(\left[\alpha_{t}^{1}, \beta_{t}^{1}\right] \times \cdots \times\left[\alpha_{t}^{n}, \beta_{t}^{n}\right]\right)$, where $\alpha_{t}^{k}=a_{i}^{k}$ and $\beta_{t}^{k}=b_{i}^{k}$ when $t_{i} \leq t<t_{i+1}$ for $i=0,1, \ldots, m-1$, and $k=1,2, \ldots, n$.

Then, for any simple function $\psi=\sum_{i=0}^{m-1} \mathbf{u}_{i} I_{\left[t_{i}, t_{i+1}\right)}$ with $\mathbf{u}_{0} \in G_{0}$, the ESM with respect to $\left(G_{t}, d_{t}^{V}\right)$ can be constructed as follows:

$$
\begin{aligned}
\phi(t)= & \pi_{G_{t}}(\psi(t))=\mathbf{u}_{0}, \quad \text { for } 0 \leq t<t_{1}, \\
\phi(t)= & \pi_{G_{t}}\left(\phi\left(t_{i-1}\right)+\psi\left(t_{i}\right)-\psi\left(t_{i-1}\right)\right), \\
& \text { for } t_{i} \leq t<t_{i+1}, i=1,2, \ldots, m-1 .
\end{aligned}
$$

The projections and the recursive construction shown above are very well known and easily verifiable. This technique has been used in $[5,9,11,15,18,20,21,23]$.

In [9], we obtained an explicit formula for the solution of the ESP on an evolving orthogonal stratum constraining system. In Theorem 2.1 of [9], we have shown that if $\left\{\left(G_{t}, d_{t}(\cdot)\right): t \geq 0\right\}$ is an orthogonal evolving stratum constraining system with $G_{t}=S\left(\left[\alpha_{t}^{1}, \beta_{t}^{1}\right] \times \cdots\right.$ $\left.\left[\alpha_{t}^{n-1}, \beta_{t}^{n-1}\right],\left[A_{t}, B_{t}\right]\right)$, then for any $\psi \in D_{G_{0}}\left([0, \infty), \mathbb{R}^{n}\right)$ the solution $(\phi, \eta)$ of the evolving $\operatorname{ESP}$ on $\left(G_{t}, d_{t}(\cdot)\right)$ is given by

$$
\begin{aligned}
\eta & =\left(-\Xi_{\alpha^{1}, \beta^{1}}\left(\psi^{1}\right),-\Xi_{\alpha^{2}, \beta^{2}}\left(\psi^{2}\right), \ldots,-\Xi_{\alpha^{n}, \beta^{n}}\left(\psi^{n}\right)\right), \\
\phi & =\psi+\eta,
\end{aligned}
$$

where

$$
\alpha_{t}^{n}=A_{t}\left(\psi^{1}(t)-\Xi_{\alpha_{t}^{1}, \beta_{t}^{1}}, \psi^{2}(t)-\Xi_{\alpha_{t}^{2}, \beta_{t}^{2}}, \ldots, \psi^{n-1}(t)-\Xi_{\alpha_{t}^{n-1}, \beta_{t}^{n-1}}\right),
$$

$\beta_{t}^{n}=B_{t}\left(\psi^{1}(t)-\Xi_{\alpha_{t}^{1}, \beta_{t}^{1}}, \psi^{2}(t)-\Xi_{\alpha_{t}^{2}, \beta_{t}^{2}}, \ldots, \psi^{n-1}(t)-\Xi_{\alpha_{t}^{n-1}, \beta_{t}^{n-1}}\right)$.

In the above, for every $i=1,2, \ldots, n$,

$$
\begin{aligned}
\Xi_{\alpha^{i}, \beta^{i}}\left(\psi^{i}\right)(t)= & I_{\left\{\tau^{\beta^{i}} \leq \tau_{\alpha^{i}}\right\}} I_{\left[\tau^{\beta^{i}}, \infty\right)} H_{\alpha^{i}, \beta^{i}}\left(\psi^{i}\right)(t) \\
& +I_{\left\{\tau_{\alpha^{i}}<\tau^{\beta^{i}}\right\}} I_{\left[\tau_{\alpha^{i}}, \infty\right]} L_{\alpha^{i}, \beta^{i}}\left(\psi^{i}\right)(t),
\end{aligned}
$$

where $I_{A}$ is an indicator function of $A$, $\tau_{\alpha}=\inf \{t>0: \alpha(t)>\psi(t)\}, \tau^{\beta}=\inf \{t>0: \psi(t)>\beta(t)\}$,

$$
\begin{aligned}
H_{\alpha, \beta}(\psi)(t) & =\sup _{0 \leq s \leq t}\left[(\psi(s)-\beta(s)) \wedge \inf _{s \leq r \leq t}(\psi(r)-\alpha(r))\right], \\
L_{\alpha, \beta}(\psi)(t) & =\inf _{0 \leq s \leq t}\left[(\psi(s)-\alpha(s)) \vee \sup _{s \leq r \leq t}(\psi(r)-\beta(r))\right] .
\end{aligned}
$$

It is our main goal here to extend the explicit formula onto much more general constraining domains by dropping the orthogonality condition.

\section{Transformations of Constraining Systems}

We now examine how the solutions of an ESP are affected by affine mappings of $\mathbb{R}^{n}$.

Proposition 1. Let $\left\{\left(G_{t}, d_{t}(\cdot)\right): t \geq 0\right\}$ be an orthogonal evolving stratum constraining system, let $T: \mathbb{R}^{n} \longrightarrow \mathbb{R}^{n}$ be an invertible affine transformation, and let $T_{0}=T-T(0)$ be its linear transformation component. For any $\psi \in$ $D_{G_{0}}\left([0, \infty), \mathbb{R}^{n}\right)$, if $(\phi, \eta)$ is a solution of ESP for $\psi$ with respect to $\left\{\left(G_{t}, d_{t}(\cdot)\right): t \geq 0\right\}$, then $\left(T \phi, T_{0} \eta\right)$ is the unique solution of ESP for $T \psi$ with respect to $\left\{\left(T G_{t}, d_{t}^{V}(\cdot)\right): t \geq 0\right\}$, where $V=T_{0}(E)$ and $E$ is the standard orthonormal basis.

Proof. Let $\psi \in D_{G_{0}}\left([0, \infty), \mathbb{R}^{n}\right)$, let $(\phi, \eta)$ be the solution of ESP for $\psi$ with respect to $\left\{\left(G_{t}, d_{t}(\cdot)\right): t \geq 0\right\}$, and let $T$ be an affine transformation. Since $\phi(t) \in G_{t}$, it follows immediately that $T \phi(t) \in T G_{t}$ for every $t \geq 0$ and so (8) holds.

Let $0 \leq s \leq t$. Since $\eta(t)-\eta(s) \in \overline{\mathrm{co}}\left[\cup_{u \in(s, t]} d_{u}(\phi(u))\right]$, we have 


$$
\begin{aligned}
T_{0} \eta(t)-T_{0} \eta(s) & =T_{0}(\eta(t)-\eta(s)) \in T_{0}\left(\overline{\mathrm{co}}\left[\cup_{u \in(s, t]} d_{u}(\phi(u))\right]\right)=\overline{\mathrm{co}}\left[T_{0}\left(\cup_{u \in(s, t]} d_{u}(\phi(u))\right)\right] \\
& =\overline{\mathrm{co}}\left[\cup_{u \in(s, t]} T_{0}\left(d_{u}(\phi(u))\right)\right]=\overline{\mathrm{co}}\left[\cup_{u \in(s, t]} d_{u}^{V}(T \phi(u))\right],
\end{aligned}
$$

where $V=T_{0}(E)$, by Remark 1 . Thus, (9) holds for every $s \in[0, t]$.

Finally, for $t>0$, we have

$$
\begin{aligned}
T_{0} \eta(t)-T_{0} \eta(t-) & =T_{0}[\eta(t)-\eta(t-)] \\
\in T_{0}\left[d_{t}(\phi(t))\right] & =T_{0}\left[d_{t}(\phi(t))\right]=d_{t}^{V}(T \phi(t)) .
\end{aligned}
$$

Thus, $\left(T \phi, T_{0} \eta\right)$ is a solution of ESP for $T \psi$. Suppose there is another solution $(\widetilde{\phi}, \widetilde{\eta})$ of ESP for $T \psi$ with respect to $\left\{\left(T G_{t}, d_{t}^{V}(\cdot)\right): t \geq 0\right\}$. Then, $\left(T^{-1} \widetilde{\phi}, T_{0}^{-1} \widetilde{\eta}\right)$ is a solution of ESP for $\psi$ with respect to $\left\{\left(G_{t}, d_{t}(\cdot)\right): t \geq 0\right\}$.

Indeed, we will show that $\left(T^{-1} \widetilde{\phi}, T_{0}^{-1} \widetilde{\eta}\right)$ satisfies conditions (8)-(10).

Since $T \psi=\widetilde{\phi}+\widetilde{\eta}$, we have $T^{-1} \widetilde{\phi}+T_{0}^{-1} \widetilde{\eta}=T_{0}^{-1}(\widetilde{\phi}-T$ $(0))+T_{0}^{-1} \widetilde{\eta}=T_{0}^{-1}(\widetilde{\phi}+\widetilde{\eta}-T(0))=T^{-1}(\widetilde{\phi}+\widetilde{\eta})=T^{-1}(T \psi)$ $=\psi$.

Since $\widetilde{\phi}(t) \in T G_{t}$, it follows that $T^{-1} \widetilde{\phi}(t) \in G_{t}$ for every $t \geq 0$ and so (8) holds.

Since $\tilde{\eta}(t)-\widetilde{\eta}(s) \in \overline{c o}\left[\cup_{u \in(s, t]} d_{u}^{V}(\tilde{\phi}(u))\right]$ for every $s \in$ $[0, t]$, we have

$$
\begin{aligned}
T_{0}^{-1} \widetilde{\eta}(t)-T_{0}^{-1} \widetilde{\eta}(s) & =T_{0}^{-1}(\widetilde{\eta}(t)-\widetilde{\eta}(s)) \in T_{0}^{-1}\left(\overline{\mathrm{co}}\left[\cup_{u \in(s, t]} d_{u}^{V}(\widetilde{\phi}(u))\right]\right) \\
& =\overline{c o}\left[\cup_{u \in(s, t]} T_{0}^{-1}\left(d_{u}^{V}(\widetilde{\phi}(u))\right)\right] \\
& =\overline{c o}\left[\cup_{u \in(s, t]} d_{u}\left(T^{-1} \widetilde{\phi}(u)\right)\right],
\end{aligned}
$$

and so (9) holds.

Finally, since $\widetilde{\eta}(t)-\widetilde{\eta}(t-) \in d_{t}^{V}(\phi(t))$, we get that $T_{0}^{-1} \widetilde{\eta}$ $(t)-T_{0}^{-1} \widetilde{\eta}(t-)=T_{0}^{-1}(\widetilde{\eta}(t)-\widetilde{\eta}(t-)) \in T_{0}^{-1}\left(d_{t}^{V}(\widetilde{\phi}(t))\right)=d_{t}$ $\left(T^{-1} \tilde{\phi}(t)\right)$, which shows that (10) holds as well. By Theorem 2.1 of [9], the solution to the evolving ESP with respect to $\left\{\left(G_{t}, d_{t}(\cdot)\right): t \geq 0\right\} \quad$ is unique. Therefore, $\left(T^{-1}\right.$ $\left.\widetilde{\phi}, T_{0}^{-1} \widetilde{\eta}\right)=(\phi, \eta)$, which means that $\left(T \phi, T_{0} \eta\right)=(\widetilde{\phi}, \widetilde{\eta})$ and so the solution $\left(T \phi, T_{0} \eta\right)$ is unique.

The above result suggests that, through the use of affine transformations, the orthogonal evolving constraining systems can generate a much larger class of constraining systems. Moreover, the affine transformation provides the link between the solutions of ESP with respect to the image constraining system and the solutions of ESP with respect to the original orthogonal constraining system.

Definition 3. A time-dependent constraining system $\left\{\left(\widetilde{G}_{t}, \widetilde{d}_{t}(\cdot)\right): t \geq 0\right\}$ in $\mathbb{R}^{n}$ is generated by an orthogonal constraining system, if there is an orthogonal evolving stratum constraining system $\left\{\left(G_{t}, d_{t}(\cdot)\right): t \geq 0\right\}$ and an affine mapping $T: \mathbb{R}^{n} \longrightarrow \mathbb{R}^{n}$ such that, for every $\psi \in$ $D_{G_{0}}\left([0, \infty), \mathbb{R}^{n}\right)$, if $(\phi, \eta)$ is the solution of ESP for $\psi$ with respect to $\left\{\left(G_{t}, d_{t}(\cdot)\right): t \geq 0\right\}$, then $\left(T \circ \phi, T_{0} \circ \eta\right)$ is the solution of ESP for $T \circ \psi$ with respect to $\left\{T\left(G_{t}\right), T_{0}\left(d_{t}\left(T_{0}^{-1}(\cdot)\right)\right): T \geq 0\right\}$. Such a mapping will be referred to as preserving the solutions of ESP.
The next results will show that every quasistratum constraining system is generated by an orthogonal constraining system.

Proposition 2. Let $\left\{\left(\widetilde{G}_{t}, \widetilde{d}_{t}\right): t \geq 0\right\}$ be an evolving quasistratum constraining system in $\mathbb{R}^{n}$, let $V$ be the associated basis as described in Definition 2, and let $T_{V}: \mathbb{R}^{n} \longrightarrow \mathbb{R}^{n}$ be the linear mapping such that $T_{V}\left(\mathbf{e}_{i}\right)=\mathbf{v}_{i}$ for $i=1,2, \ldots, n$. Then, $\left\{\left(\widetilde{G}_{t}, \widetilde{d}_{t}\right): t \geq 0\right\}$ is generated by an orthogonal evolving stratum constraining system and $T_{V}$ is preserving the solutions of the ESP.

Proof. By Definition 2, there are functions $\alpha^{i} \leq$ $\beta^{i}, i=1,2, \ldots, n-1$, and $A \leq B$ such that, for every $t \geq 0$, $\widetilde{G}_{t}=G_{t}^{V}=S^{V}\left(\left[\alpha_{t}^{1}, \beta_{t}^{1}\right] \times \cdots \times\left[\alpha_{t}^{n-1}, \beta_{t}^{n-1}\right],\left[A_{t}, B_{t}\right]\right) \quad$ and $\tilde{d}_{t}(\mathbf{x})=d_{t}^{V}(\mathbf{x})$ as defined in (4).

Let $G_{t}=S\left(\left[\alpha_{t}^{1}, \beta_{t}^{1}\right] \times \cdots \times\left[\alpha_{t}^{n-1}, \beta_{t}^{n-1}\right],\left[A_{t}, B_{t}\right]\right)$ and let $d_{t}=d_{t}^{E}$ be the associated orthogonal constraining field. Then, $\widetilde{G}_{t}=T_{V}\left(G_{t}\right)$ and $d_{t}^{V}(\mathbf{x})=T_{V}\left(d_{t}\left(T_{V}^{-1}(\mathbf{x})\right)\right)$ for every $\mathbf{x}$, as seen in Remark 1.

\section{Explicit Solutions of ESP}

The relationship between the solutions of the ESP on an evolving orthogonal constraining system $\left(G_{t}, d_{t}\right)$ and the corresponding solutions of the ESP on a constraining system $\left(\widetilde{G}_{t}, \widetilde{d}_{t}\right)$ generated by it as described in Propositions 1 and 2 permits now an extension of the explicit formulas (15).

Theorem 1. Let $(\widetilde{G}, \widetilde{d})$ be an evolving quasistratum constraining system in $\mathbb{R}^{n}$; that is, there is a basis $V=\left\{\mathbf{v}_{1}, \mathbf{v}_{1}, \ldots, \mathbf{v}_{n}\right\}, \quad$ such that $\widetilde{G}_{t}=S^{V}\left(\left[\alpha_{t}^{1}, \beta_{t}^{1}\right] \times \ldots\right.$ $\left.\times\left[\alpha_{t}^{n-1}, \beta_{t}^{n-1}\right],\left[A_{t}, B_{t}\right]\right)$ and $\widetilde{d}_{t}=d_{t}^{V}$, for every $t \geq 0$. Then, for any $\widetilde{\psi} \in D_{\widetilde{G}}\left([0, \infty), \mathbb{R}^{n}\right)$, the evolving ESP on $(\widetilde{G}, \widetilde{d})$ has the unique solution $(\tilde{\phi}, \widetilde{\eta})$ given by

$$
\begin{aligned}
& \tilde{\eta}=T_{V}\left(-\Xi_{\alpha^{1}, \beta^{1}}\left(\psi^{1}\right),-\Xi_{\alpha^{2}, \beta^{2}}\left(\psi^{2}\right), \ldots,-\Xi_{\alpha^{n}, \beta^{n}}\left(\psi^{n}\right)\right), \\
& \tilde{\phi}=\widetilde{\psi}+\widetilde{\eta},
\end{aligned}
$$

where $T_{V}: \mathbb{R}^{n} \longrightarrow \mathbb{R}^{n}$ is the linear transformation defined by $T_{V}\left(\mathbf{e}_{i}\right)=\mathbf{v}_{i}$ for every $i=1,2, \ldots, n$,

$$
\begin{aligned}
\psi(t) & =\left(\psi^{1}(t), \psi^{2}(t), \ldots, \psi^{n}(t)\right)=\left(T_{V}\right)^{-1} \\
& \left(\left(\widetilde{\psi}^{1}(t), \widetilde{\psi}^{2}(t), \ldots, \widetilde{\psi}^{n}(t)\right)_{V}\right)=\left(T_{V}\right)^{-1}(\widetilde{\psi}(t)), \\
\alpha_{t}^{n} & =A_{t}\left(\psi^{1}(t)-\Xi_{\alpha_{t}^{1}, \beta_{t}^{1}}, \psi^{2}(t)-\Xi_{\alpha_{t}^{2}, \beta_{t}^{2}}, \ldots, \psi^{n-1}(t)-\Xi_{\alpha_{t}^{n-1}, \beta_{t}^{n-1}}\right),
\end{aligned}
$$

$\beta_{t}^{n}=B_{t}\left(\psi^{1}(t)-\Xi_{\alpha_{t}^{1}, \beta_{t}^{1}}, \psi^{2}(t)-\Xi_{\alpha_{t}^{2}, \beta_{t}^{2}}, \ldots, \psi^{n-1}(t)-\Xi_{\alpha_{t}^{n-1}, \beta_{t}^{n-1}}\right)$. 
In the above, for every $i=1,2, \ldots, n$,

$$
\begin{aligned}
\Xi_{\alpha^{i}, \beta^{i}}\left(\psi^{i}\right)(t)= & I_{\left\{\tau^{\beta^{i}} \leq \tau_{\alpha^{i}}\right\}} I_{\left[\tau^{\beta^{i}}, \infty\right)} H_{\alpha^{i}, \beta^{i}}\left(\psi^{i}\right)(t) \\
& +I_{\left\{\tau_{\alpha^{i}}<\tau^{\beta^{i}}\right\}} I_{\left[\tau_{\alpha^{i}}, \infty\right)} L_{\alpha^{i}, \beta^{i}}\left(\psi^{i}\right)(t),
\end{aligned}
$$

where $\tau_{\alpha^{i}}=\inf \left\{t>0 \mid \alpha^{i}(t)-\psi^{i}(t)>0\right\}, \quad \tau^{\beta^{i}}=\inf \left\{t>0 \mid \psi^{i}\right.$ $\left.(t)-\beta^{i}(t)>0\right\}$, while

$$
\begin{aligned}
H_{\alpha, \beta}(\psi)(t) & =\sup _{0 \leq s \leq t}\left[(\psi(s)-\beta(s)) \wedge \inf _{s \leq r \leq t}(\psi(r)-\alpha(r))\right], \\
L_{\alpha, \beta}(\psi)(t) & =\inf _{0 \leq s \leq t}\left[(\psi(s)-\alpha(s)) \vee \sup _{s \leq r \leq t}(\psi(r)-\beta(r))\right] .
\end{aligned}
$$

Proof. Let $(\widetilde{G}, \widetilde{d})$ be an evolving quasistratum constraining system in $\mathbb{R}^{n}$. By Proposition 2 , there is a basis $V$ of $\mathbb{R}^{n}$ such that $\widetilde{G}_{t}=T_{V}\left(G_{t}\right)$ and $\widetilde{d}_{t}(\mathbf{x})=d_{t}^{V}(\mathbf{x})=T_{V}\left(d_{t}\left(T_{V}^{-1}(\mathbf{x})\right)\right)$, where $\left(G_{t}, d_{t}\right)$ is an orthogonal stratum constraining system having a representation $S\left(\left[\alpha_{t}^{1}, \beta_{t}^{1}\right] \times \cdots \times \quad\left[\alpha_{t}^{n-1}\right.\right.$, $\left.\left.\beta_{t}^{n-1}\right],\left[A_{t}, B_{t}\right]\right)$. Let $\quad \psi(t)=\left(\psi^{1}(t), \psi^{2}(t), \ldots, \psi^{n}(t)\right)=$ $T_{V}^{-1}(\widetilde{\psi}(t))$. Then, $\psi \in D_{G_{0}}\left([0, \infty), \mathbb{R}^{n}\right)$ and by Theorem 2.1 in [9], as shown in (15), the ESP for $\psi$ on $(G, d)$ has a unique solution $(\phi, \eta)$ given by

$$
\begin{aligned}
& \eta=\left(-\Xi_{\alpha^{1}, \beta^{1}}\left(\psi^{1}\right),-\Xi_{\alpha^{2}, \beta^{2}}\left(\psi^{2}\right), \ldots,-\Xi_{\alpha^{n}, \beta^{n}}\left(\psi^{n}\right)\right), \\
& \phi=\psi+\eta,
\end{aligned}
$$

where $\alpha^{n}, \beta^{n}$ are defined by (16), (17), and $\Xi_{\alpha^{i}, \beta^{i}}$ is defined by (18) for $i=1,2, \ldots, n$. Hence, by Proposition $1,\left(T_{V} \phi, T_{V} \eta\right)$ is a solution of ESP for $\widetilde{\psi}$ on $(\widetilde{G}, \widetilde{d})$. The uniqueness of $(\widetilde{\phi}, \widetilde{\eta})$ follows immediately from the uniqueness of $(\phi, \eta)$.

\section{Lipschitz Properties}

Lipschitz continuity is the most desirable property of the ESM. Unfortunately, establishing the best Lipschitz conditions tends to get very technical. In this paper, the discussion of this property will be limited to quasiblocks under two different norms.

Given any invertible linear operator $A: \mathbb{R}^{n} \longrightarrow \mathbb{R}^{n}$ and the associated basis $V=\left\{\mathbf{v}_{1}, \mathbf{v}_{1}, \ldots, \mathbf{v}_{n}\right\}$, where $\mathbf{v}_{i}=$ $A\left(\mathbf{e}_{i}\right), i=1,2, \ldots, n$, we define $\mathrm{a}_{V}$ norm on $\mathbb{R}^{n}$ by

$$
\|\mathbf{x}\|_{V}=\left\|A^{-1}(\mathbf{x})\right\|
$$

Note that $\left\|x^{1} \mathbf{v}_{1}+x^{2} \mathbf{v}_{2}+\cdots+x^{n} \mathbf{v}_{n}\right\|_{V}=\| x^{1} \mathbf{e}_{1}+x^{2} \mathbf{e}_{2}+$ $\cdots+x^{n} \mathbf{e}_{n} \|$. This norm induces a corresponding norm on $D\left([0, \infty), \mathbb{R}^{n}\right)$ defined by

$$
\|\psi\|_{V}=\sup _{t \geq 0}\|\psi(t)\|_{V}, \quad \text { for } \psi \in D\left([0, \infty), \mathbb{R}^{n}\right) .
$$

Some easily obtainable Lipschitz conditions for solutions of the ESP on evolving quasistratum and quasiblock constraining systems are inherited from the evolving orthogonal stratum or block constraining systems via the linear transformation.

Proposition 3. Let $(\widetilde{G}, \widetilde{d})$ be an evolving quasiblock constraining system in $\mathbb{R}^{n}$ that can be represented as an image of an orthogonal evolving block constraining system via an invertible linear transformation $T_{V}$, where $V$ is the image of the orthonormal basis through $T_{V}$. If $\left(\widetilde{\phi}_{1}, \widetilde{\eta}_{1}\right)$ and $\left(\widetilde{\phi}_{2}, \widetilde{\eta}_{2}\right)$ are the solutions of the ESP for $\widetilde{\psi}_{1}$ and $\widetilde{\psi}_{2}$ with respect to $(\widetilde{G}, \widetilde{d})$, then the following Lipschitz conditions hold:

$$
\begin{aligned}
& \left\|\widetilde{\eta}_{1}-\widetilde{\eta}_{2}\right\|_{V} \leq \sqrt{n}\left\|\widetilde{\psi}_{1}-\widetilde{\psi}_{2}\right\|_{V}, \\
& \left\|\widetilde{\phi}_{1}-\widetilde{\phi}_{2}\right\|_{V} \leq(1+\sqrt{n})\left\|\widetilde{\psi}_{1}-\widetilde{\psi}_{2}\right\|_{V} .
\end{aligned}
$$

Proof. Let $(\widetilde{G}, \widetilde{d})$ be an evolving quasiblock constraining system in $\mathbb{R}^{n}$. Then, by Proposition 2, there is a basis $V$ and an evolving block constraining system $(G, d)$ whose image through $T_{V}$ is $(\tilde{G}, \tilde{d})$. Let $\psi_{1}=T_{V}^{-1} \circ \widetilde{\psi}_{1}$ and $\psi_{2}=T_{V}^{-1} \circ \widetilde{\psi}_{2}$. Then, by Proposition 1, the solutions of the ESP for $\psi_{1}$ and $\psi_{2}$ with respect to $(G, d)$ are $\left(\phi_{1}, \eta_{1}\right)$ and $\left(\phi_{2}, \eta_{2}\right)$, where $\phi_{i}=T_{V}^{-1} \circ \widetilde{\phi}_{i}, \eta_{i}=T_{V}^{-1} \circ \widetilde{\eta}_{i}$ for $i=1,2$. By Proposition 3.1 in [9],

$$
\left\|\eta_{1}-\eta_{2}\right\| \leq \sqrt{n}\left\|\psi_{1}-\psi_{2}\right\|
$$

Therefore,

$$
\begin{aligned}
\left\|\widetilde{\eta}_{1}-\widetilde{\eta}_{2}\right\|_{V} & =\sup _{t \geq 0}\left\|\widetilde{\eta}_{1}(t)-\widetilde{\eta}_{2}(t)\right\|_{V}=\sup _{t \geq 0}\left\|T_{V}\left(\eta_{1}(t)\right)-T_{V}\left(\eta_{2}(t)\right)\right\|_{V}=\sup _{t \geq 0}\left\|T_{V}\left(\eta_{1}(t)-\eta_{2}(t)\right)\right\|_{V} \\
& =\sup _{t \geq 0}\left\|\eta_{1}(t)-\eta_{2}(t)\right\|=\left\|\eta_{1}-\eta_{2}\right\| \leq \sqrt{n}\left\|\psi_{1}-\psi_{2}\right\|=\sqrt{n} \sup _{t \geq 0}\left\|\psi_{1}(t)-\psi_{2}(t)\right\| \\
& =\sqrt{n} \sup _{t \geq 0}\|\| T_{V}\left(\psi_{1}(t)-\psi_{2}(t)\right)\left\|_{V}=\sqrt{n} \sup _{t \geq 0}\right\| T_{V}\left(\psi_{1}(t)\right)-T_{V}\left(\psi_{2}(t)\right) \|_{V} \\
& =\sqrt{n} \sup _{t \geq 0}\left\|\widetilde{\psi}_{1}(t)-\widetilde{\psi}_{2}(t)\right\|_{V}=\sqrt{n}\left\|\widetilde{\psi}_{1}-\widetilde{\psi}_{2}\right\|_{V},
\end{aligned}
$$


and so (32) is proven. The Lipschitz condition (33) follows now immediately via the triangle inequality:

$$
\begin{aligned}
\left\|\widetilde{\phi}_{1}-\widetilde{\phi}_{2}\right\|_{V} & =\left\|\widetilde{\psi}_{1}-\widetilde{\psi}_{2}+\widetilde{\eta}_{1}-\widetilde{\eta}_{2}\right\|_{V} \\
& \leq\left\|\widetilde{\psi}_{1}-\widetilde{\psi}_{2}\right\|_{V}+\left\|\widetilde{\eta}_{1}-\widetilde{\eta}_{2}\right\|_{V}=(1+\sqrt{n})\left\|\widetilde{\psi}_{1}-\widetilde{\psi}_{2}\right\|_{V} .
\end{aligned}
$$

The Lipschitz constants in Proposition 3 are tight as the Lipschitz constant in Proposition 3.1 in [9] is tight.

More useful for applications of the ESM is Lipschitz continuity in the standard norm on $D\left([0, \infty), \mathbb{R}^{n}\right)$ generated by the Euclidean norm on $\mathbb{R}^{n}$.

We shall use $\|T\|$ to denote the norm of $T$ as a linear operator. Thus,

$$
\|T\|=\sup _{\|\mathbf{x}\| \leq 1}\|T \mathbf{x}\|=\inf \{K:\|T \mathbf{x}\| \leq K\|\mathbf{x}\| \text { for all } \mathbf{x}\} .
$$

Remark 2. Let $T: \mathbb{R}^{n} \longrightarrow \mathbb{R}^{n}$ be a linear transformation such that $T \mathbf{e}_{i}=\mathbf{v}_{i}, i=1,2, \ldots, n$. Then, for every $\mathbf{x}=\left(x_{1}, x_{2}, \ldots, x_{n}\right) \in \mathbb{R}^{n}$,

$$
\left\|\sum_{i=1}^{n} x^{i} \mathbf{v}_{i}\right\| \leq\|T\|\|\mathbf{x}\| .
$$

Proof

$$
\left\|\sum_{i=1}^{n} x^{i} \mathbf{v}_{i}\right\|=\left\|\sum_{i=1}^{n} x^{i} T\left(\mathbf{e}_{i}\right)\right\|=\|T \mathbf{x}\| \leq\|T\|\|x\| .
$$

One easy way to obtain a Lipschitz constant for the ESM is to use the operator norms of $T_{V}$ and $T_{V}^{-1}$.

Proposition 4. Let $(\widetilde{G}, \widetilde{d})$ be an evolving quasiblock constraining system in $\mathbb{R}^{n}$ that can be represented as an image of an orthogonal block constraining system via an invertible linear transformation $T$ and let $V$ be the image of the orthonormal basis through T. If $\left(\widetilde{\phi}_{1}, \widetilde{\eta}_{1}\right)$ and $\left(\widetilde{\phi}_{2}, \widetilde{\eta}_{2}\right)$ are the solutions of the ESP for $\widetilde{\psi}_{1}$ and $\tilde{\psi}_{2}$ with respect to $(\tilde{G}, \tilde{d})$, then the following Lipschitz conditions hold:

$$
\begin{aligned}
& \left\|\widetilde{\eta}_{1}-\widetilde{\eta}_{2}\right\| \leq \sqrt{n} \cdot\|T\| \cdot\left\|T^{-1}\right\| \cdot\left\|\widetilde{\psi}_{1}-\widetilde{\psi}_{2}\right\|, \\
& \left\|\widetilde{\phi}_{1}-\widetilde{\phi}_{2}\right\| \leq\left(1+\sqrt{n} \cdot\|T\| \cdot\left\|T^{-1}\right\|\right)\left\|\widetilde{\psi}_{1}-\widetilde{\psi}_{2}\right\| .
\end{aligned}
$$

Proof. Let $\psi_{i}=T^{-1} \widetilde{\psi}_{i}, \quad \phi_{i}=T^{-1} \widetilde{\phi}_{i}$, and $\eta_{i}=T^{-1} \widetilde{\eta}_{i}$ for $i=1,2$. For every $t \geq 0$, by Proposition 3.1 in [9],

$$
\begin{aligned}
\left\|\widetilde{\eta}_{1}(t)-\widetilde{\eta}_{2}(t)\right\| & =\left\|T \eta_{1}(t)-T \eta_{2}(t)\right\|=\left\|T\left(\eta_{1}(t)-\eta_{2}(t)\right)\right\| \leq\|T\| \cdot\left\|\eta_{1}(t)-\eta_{2}(t)\right\| \\
& \leq\|T\| \cdot \sqrt{n} \cdot \sup _{0 \leq t \leq \infty}\left\|\psi_{1}(t)-\psi_{2}(t)\right\|=\|T\| \cdot \sqrt{n} \cdot \sup _{0 \leq t \leq \infty}\left\|T^{-1}\left(\widetilde{\psi}_{1}(t)-\widetilde{\psi}_{2}(t)\right)\right\| \\
& \leq \sqrt{n} \cdot\|T\| \cdot\left\|T^{-1}\right\| \sup _{0 \leq t \leq \infty}\left\|\widetilde{\psi}_{1}(t)-\widetilde{\psi}_{2}(t)\right\|=\sqrt{n} \cdot\|T\| \cdot\left\|T^{-1}\right\| \cdot\left\|\widetilde{\psi}_{1}-\widetilde{\psi}_{2}\right\|,
\end{aligned}
$$

which ends the proof of (40). Now, we obtain (41) by triangle

inequality. Indeed, for any $t \geq 0$,

$$
\begin{aligned}
\left\|\widetilde{\phi}_{1}(t)-\widetilde{\phi}_{2}(t)\right\| & \leq\left\|\widetilde{\eta}_{1}(t)-\tilde{\eta}_{2}(t)\right\|+\left\|\widetilde{\psi}_{1}(t)-\widetilde{\psi}_{2}(t)\right\| \leq \sqrt{n} \cdot\|T\| \cdot\left\|T^{-1}\right\| \cdot\left\|\widetilde{\psi}_{1}-\widetilde{\psi}_{2}\right\|+\left\|\widetilde{\psi}_{1}-\widetilde{\psi}_{2}\right\| \\
& \leq\left(1+\sqrt{n} \cdot\|T\| \cdot\left\|T^{-1}\right\|\right)\left\|\widetilde{\psi}_{1}-\widetilde{\psi}_{2}\right\|,
\end{aligned}
$$

ending the proof of (41).

The Lipschitz constants in Proposition 4 are not tight as will be seen in Example 3. Unfortunately, the author's attempts to derive the best constants seem to produce inadvertently very technical results. The Lipschitz constant derived for the ESM with an orthogonal evolving stratum constraining system in Theorem 4.1 of [9] was tight but it was not simple and rather technical to derive. That is why the results in this section are limited to quasiblock restraining systems. Still, we are going to improve the results of Proposition 4 and produce smaller constants.

We shall need some technical inequalities involving vectors and angles between vectors. We will use $\angle(\mathbf{x}, \mathbf{y})$ to denote the smallest angle between vectors $\mathbf{x}$ and $\mathbf{y}$. Thus, if $\angle(\mathbf{x}, \mathbf{y})=\theta$, then $0 \leq \theta \leq \pi$.
Lemma 1. If $\mathbf{x}, \mathbf{y} \in \mathbb{R}^{n}$ and $\angle(\mathbf{x}, \mathbf{y})=\theta$, where $0<\theta<\pi$, then

$$
\|\mathbf{x}-\mathbf{y}\| \leq \tan \frac{\theta}{2} \cdot\|\mathbf{x}+\mathbf{y}\|
$$

Furthermore, if $\|\mathbf{x}\|=\|\mathbf{y}\|$, then

$$
\|\mathbf{x}-\mathbf{y}\|=\tan \frac{\theta}{2} \cdot\|\mathbf{x}+\mathbf{y}\| .
$$

Proof. Because the two independent vectors span a twodimensional space, it is enough to prove (44) in $\mathbb{R}^{2}$. We can also assume without loss of generality that $\mathbf{x}=(r, 0)$ for some $r>0$ and $\mathbf{y}=(\cos \theta, \sin \theta)$, where $0<\theta<\pi$. Then, $\|\mathbf{x}+\mathbf{y}\|^{2}=\|(r+\cos \theta, \sin \theta)\|^{2}=r^{2}+2 r \cos \theta+1$ and 
$\|\mathbf{x}-\mathbf{y}\|^{2}=\|(r-\cos \theta,-\sin \theta)\|^{2}=r^{2}-2 r \cos \theta+1$. We define a function $g:(0, \infty) \longrightarrow(0, \infty)$ by

$$
g(r)=\frac{\|\mathbf{x}-\mathbf{y}\|^{2}}{\|\mathbf{x}+\mathbf{y}\|^{2}}=\frac{r^{2}-2 r \cos \theta+1}{r^{2}+2 r \cos \theta+1} .
$$

A quick analysis of its derivative

$$
g^{\prime}(r)=\frac{4 \cos \theta\left(r^{2}-1\right)}{\left(r^{2}+2 r \cos \theta+1\right)^{2}}
$$

shows that the maximum value of $g(r)$ occurs when $r=1$ and $g(1)=\tan ^{2}(\theta / 2)$. Since $0<\theta<\pi$, $\tan (\theta / 2)>0$, and therefore,

$$
\max \left\{\frac{\|\mathbf{x}-\mathbf{y}\|}{\|\mathbf{x}+\mathbf{y}\|}: \angle(\mathbf{x}, \mathbf{y})=\theta\right\}=\sqrt{g(1)}=\tan \frac{\theta}{2} .
$$

We need to introduce now some necessary notations. Let $\Lambda$ be the set of all permutations $\lambda:\{1,2, \ldots$, $n\} \longrightarrow\{1,2, \ldots, n\}$. Given any sequence of $n$ independent vectors $\mathbf{v}_{1}, \mathbf{v}_{2}, \ldots, \mathbf{v}_{n}$ and any $\lambda \in \Lambda$, we define

$$
\begin{aligned}
& C_{k}^{\lambda}(V)=\tan \left(\frac{1}{2} \angle\left(\sum_{i=1}^{k} \mathbf{v}_{\lambda(i)}, \sum_{i=k+1}^{n} \mathbf{v}_{\lambda(i)}\right)\right), \\
& \text { for } k=1,2, \ldots, n-1, \\
& C_{k}^{\lambda}(V)=1, \quad \text { for } k=n .
\end{aligned}
$$

Finally, we set

$$
C_{V}=\max _{1 \leq k \leq n} \max _{\lambda \in \Lambda} C_{k}^{\lambda}(V)
$$

Lemma 2. For any sequence $\mathbf{v}_{1}, \mathbf{v}_{2}, \ldots, \mathbf{v}_{n}$ of independent vectors in $\mathbb{R}^{n}$,

$$
\max \left\{\left\|\sum_{i=1}^{n} t_{i} \mathbf{v}_{i}\right\|:-1 \leq t_{i} \leq 1, i=1,2, \ldots, n\right\}=C_{V}\left\|\sum_{i=1}^{n} \mathbf{v}_{i}\right\| .
$$

Proof. Let $g:[-1,1]^{n} \longrightarrow[0, \infty)$ be a function defined by $g\left(t_{1}, t_{2}, \ldots, t_{n}\right)=\left\|\sum_{i=1}^{n} t_{i} \mathbf{v}_{i}\right\|$. Then, $g$ is a convex nonnegative function and it attains maximum value at some extreme point of $[-1,1]^{n}$; that is, there are $n$ values $\varepsilon^{1}, \varepsilon^{2}, \ldots, \varepsilon^{n}$ such that $\varepsilon^{i} \in\{-1,1\}$ for $i=1,2, \ldots, n$ and

$\max \left\{g\left(t_{1}, t_{2}, \ldots, t_{n}\right): t_{i} \in[-1,1], i=1,2, \ldots, n\right\}=g\left(\varepsilon^{1}, \varepsilon^{2}, \ldots, \varepsilon^{n}\right)$.

Let $k$ be the number of coordinates of $\left(\varepsilon^{1}, \varepsilon^{2}, \ldots, \varepsilon^{n}\right)$ that are positive and let $\lambda$ be a permutation in $\Lambda$ such that $\varepsilon^{\lambda(1)}=$ $\varepsilon^{\lambda(2)}=\cdots=\varepsilon^{\lambda(k)}=1$ while $\varepsilon^{\lambda(i)}=-1$ for $i=k+1, \ldots, n$. Then, by Lemma 1 ,

$$
\left\|\sum_{i=1}^{n} \varepsilon^{\lambda(i)} \mathbf{v}_{\lambda(i)}\right\|=\left\|\sum_{i=1}^{k} \mathbf{v}_{\lambda(i)}-\sum_{i=k+1}^{n} \mathbf{v}_{\lambda(i)}\right\| \leq C_{k}^{\lambda}(V)\left\|\sum_{i=1}^{n} \mathbf{v}_{\lambda(i)}\right\| \leq C(V)\left\|\sum_{i=1}^{n} \mathbf{v}_{i}\right\| .
$$

Lemma 3. Let $\mathbf{x}, \mathbf{y}$ be two independent vectors in $\mathbb{R}^{n}$ and let $\angle(\mathbf{x}, \mathbf{y})=\theta$. Then,

$$
\max \{\|\mathbf{x}\|,\|\mathbf{y}\|\} \leq \frac{1}{\sin (\theta)}\|\mathbf{x}+\mathbf{y}\| .
$$

Proof. We can assume without loss of generality that $n=2$. Consider the parallelogram with vertices at $0, \mathbf{x}, \mathbf{y}$ and $\mathbf{x}+\mathbf{y}$. In any parallelogram, the distance between the parallel lines through the opposite sides cannot exceed the length of each diagonal. The distance from $\mathbf{x}$ to the line through 0 and $\mathbf{y}$ is $\|\mathbf{x}\| \sin \theta$ and so it cannot exceed $\|\mathbf{x}+\mathbf{y}\|$. Similarly, the distance from $\mathbf{y}$ to the line through 0 and $\mathbf{x}$ is $\|\mathbf{y}\| \sin \theta$ and it cannot exceed $\|\mathbf{x}+\mathbf{y}\|$. Therefore, $\|\mathbf{x}\| \leq(\|\mathbf{x}+\mathbf{y}\| / \sin \theta)$ and $\|\mathbf{y}\| \leq(\|\mathbf{x}+\mathbf{y}\| / \sin \theta)$.

Given a sequence of $n$ independent vectors $V=\left\{\mathbf{v}_{1}, \mathbf{v}_{2}, \ldots, \mathbf{v}_{n}\right\}$, let $V_{j}$ denote the linear subspace spanned by vectors $V \backslash\left\{\mathbf{v}_{j}\right\}$, let $K_{j}=\sin \angle\left(\mathbf{v}_{j}, V_{j}\right)$, and let $K_{V}=\min _{1 \leq j \leq n} K_{j}$. It will be also convenient to use the following notations: $\overline{\mathbf{v}}=(\mathbf{v} /\|\mathbf{v}\|)$ and $\bar{V}=\left\{\overline{\mathbf{v}}_{1}, \overline{\mathbf{v}}_{2}, \ldots, \overline{\mathbf{v}}_{n}\right\}$. It is important to notice two things. First,

$$
K_{V} \leq K_{j}=\sin \left(\angle\left(\mathbf{v}_{j}, V_{j}\right)\right) \leq \sin \left(\angle\left(\mathbf{v}_{j}, \sum_{i=1}^{n} \mathbf{v}_{i}-v_{j}\right)\right)
$$

for every $j=1,2, \ldots, n$.

Second, $K_{V}$ depends only on the directions of vectors in $V$ and not on their magnitudes, that is,

$$
K_{V}=K_{\bar{V}} \text {. }
$$

Lemma 4. Let $\mathbf{v}_{1}, \mathbf{v}_{2}, \ldots, \mathbf{v}_{\mathbf{n}}$ be independent vectors in $\mathbb{R}^{n}$. Then, for every $j=1,2, \ldots, n$,

$$
\left\|\mathbf{v}_{\mathbf{j}}\right\| \leq \frac{1}{K_{\bar{V}}}\left\|\sum_{i=1}^{n} \mathbf{v}_{\mathbf{i}}\right\| .
$$

Proof. Let $j \in\{1,2, \ldots, n\}$. Applying Lemma 3 to vectors $\mathbf{v}_{\mathbf{j}}$ and $\sum_{i=1}^{n} \mathbf{v}_{i}-\mathbf{v}_{j}$ and using (55) and (56), we can see that

$$
\left\|\mathbf{v}_{\mathbf{j}}\right\| \leq \frac{1}{K_{j}}\left\|\mathbf{v}_{\mathbf{j}}+\sum_{i=1}^{n} \mathbf{v}_{\mathbf{i}}-\mathbf{v}_{\mathbf{j}}\right\| \leq \frac{1}{K_{\bar{V}}}\left\|\sum_{i=1}^{n} \mathbf{v}_{\mathbf{i}}\right\| .
$$

Theorem 2. Let $(\widetilde{G}, \widetilde{d})$ be an evolving quasiblock constraining system in $\mathbb{R}^{n}$ that can be represented as an image of an orthogonal evolving block constraining system via an invertible linear transformation $T$ and let $V$ be the image of the orthonormal basis through T. If $\left(\widetilde{\phi}_{1}, \widetilde{\eta}_{1}\right)$ and $\left(\widetilde{\phi}_{2}, \widetilde{\eta}_{2}\right)$ are the solutions of the ESP for $\widetilde{\psi}_{1}$ and $\widetilde{\psi}_{2}$ with respect to $(\widetilde{G}, \widetilde{d})$, then the following Lipschitz conditions hold

$$
\begin{aligned}
& \left\|\widetilde{\eta}_{1}-\widetilde{\eta}_{2}\right\| \leq L_{V} \cdot\left\|\widetilde{\psi}_{1}-\widetilde{\psi}_{2}\right\|, \\
& \left\|\widetilde{\phi}_{1}-\widetilde{\phi}_{2}\right\| \leq\left(1+L_{V}\right)\left\|\widetilde{\psi}_{1}-\widetilde{\psi}_{2}\right\|,
\end{aligned}
$$

where 


$$
L_{V}=\frac{C_{\bar{V}}}{K_{\bar{V}}}\left\|\sum_{i=1}^{n} \overline{\mathbf{v}}_{i}\right\| .
$$

Proof. For any $t \geq 0$,

$$
\begin{aligned}
\left\|\widetilde{\eta}_{1}(t)-\tilde{\eta}_{2}(t)\right\| & =\left\|\sum_{i=1}^{n}\left(\eta_{1}^{i}(t)-\eta_{2}^{i}(t)\right) \mathbf{v}_{i}\right\| \\
& =\left\|\sum_{i=1}^{n}\left(\eta_{1}^{i}(t)-\eta_{2}^{i}(t)\right)\right\| \mathbf{v}_{i}\left\|\cdot \frac{\mathbf{v}_{i}}{\left\|\mathbf{v}_{i}\right\|}\right\|,
\end{aligned}
$$

where, by (3.2) of [9], for every $i=1,2, \ldots, n$,

$$
\left|\eta_{1}^{i}(t)-\eta_{2}^{i}(t)\right| \cdot\left\|\mathbf{v}_{i}\right\| \leq \max _{1 \leq i \leq n} \max _{0 \leq s \leq t}\left|\psi_{1}^{i}(s)-\psi_{2}^{i}(s)\right| \cdot\left\|\mathbf{v}_{i}\right\| .
$$

Thus, by Lemma 2,

$$
\begin{aligned}
\left\|\widetilde{\eta}_{1}(t)-\widetilde{\eta}_{2}(t)\right\| & \leq \max _{1 \leq i \leq n} \max _{0 \leq s \leq t}\left(\left|\psi_{1}^{i}(s)-\psi_{2}^{i}(s)\right| \cdot\left\|\mathbf{v}_{i}\right\|\right) \cdot C_{\bar{V}} \cdot\left\|\sum_{i=1}^{n} \frac{\mathbf{v}_{i}}{\left\|\mathbf{v}_{i}\right\|}\right\| \\
& =\max _{0 \leq s \leq t}\left[\max _{1 \leq i \leq n}\left(\left|\psi_{1}^{i}(s)-\psi_{2}^{i}(s)\right| \cdot\left\|\mathbf{v}_{i}\right\|\right)\right] \cdot C_{\bar{V}} \cdot\left\|\sum_{i=1}^{n} \overline{\mathbf{v}}_{i}\right\| .
\end{aligned}
$$

By Lemma 4, for every $0 \leq s \leq t$,

$$
\begin{aligned}
\max _{1 \leq i \leq n}\left(\left|\psi_{1}^{i}(s)-\psi_{2}^{i}(s)\right| \cdot\left\|\mathbf{v}_{i}\right\|\right) & \leq \frac{1}{K_{\bar{V}}}\left\|\sum_{i=1}^{n}\left[\psi_{1}^{i}(s)-\psi_{2}^{i}(s)\right] \mathbf{v}_{i}\right\| \\
& =\frac{1}{K_{\bar{V}}}\left\|\widetilde{\psi}_{1}(s)-\widetilde{\psi}_{2}(s)\right\| .
\end{aligned}
$$

Therefore, for every $t \geq 0$,

$$
\begin{aligned}
\left\|\widetilde{\eta}_{1}(t)-\widetilde{\eta}_{2}(t)\right\| & \leq \max _{0 \leq s \leq t} \frac{C_{\bar{V}}}{K_{\bar{V}}}\left\|\sum_{i=1}^{n} \overline{\mathbf{v}}_{i}\right\| \cdot\left\|\widetilde{\psi}_{1}(s)-\widetilde{\psi}_{2}(s)\right\| \\
& =\frac{C_{\bar{V}}}{K_{\bar{V}}}\left\|\sum_{i=1}^{n} \overline{\mathbf{v}}_{i}\right\| \cdot\left\|\widetilde{\psi}_{1}-\widetilde{\psi}_{2}\right\|,
\end{aligned}
$$

which ends the proof of (59).

To prove (60), we use (59) and the triangle inequality:

$$
\begin{aligned}
\left\|\widetilde{\phi}_{1}-\tilde{\phi}_{2}\right\| & =\left\|\widetilde{\psi}_{1}-\widetilde{\psi}_{2}+\tilde{\eta}_{1}-\widetilde{\eta}_{2}\right\| \leq\left\|\widetilde{\psi}_{1}-\widetilde{\psi}_{2}\right\|+\left\|\tilde{\eta}_{1}-\widetilde{\eta}_{2}\right\| \\
& \leq\left(1+\frac{C_{\bar{V}}}{K_{\bar{V}}}\left\|\sum_{i=1}^{n} \overline{\mathbf{v}}_{i}\right\|\right)\left\|\widetilde{\psi}_{1}-\widetilde{\psi}_{2}\right\| .
\end{aligned}
$$

Remark 3. The Lipschitz constants of Theorem 2 depend only on the angles defining the shape of the quasiblock and not on its size.

Remark 4. In the special case of an orthogonal block constraining system, all the relevant angles are right angles and therefore $C_{\bar{V}}=1$ and $K_{\bar{V}}=1$. Thus, the Lipschitz constant in
(59) becomes $\left\|\sum_{i=1}^{n} \overline{\mathbf{v}}_{i}\right\|=\sqrt{n}$ matching the result of Proposition 3.1 in [9].

The following example will show that the Lipschitz constant $L_{V}$ in (59) is tight in $\mathbb{R}^{2}$. Essentially, it will demonstrate that for any quasiblock constraining system in $\mathbb{R}^{2}$, there are functions $\psi_{1}$ and $\psi_{2}$ such that $\left\|\eta_{1}-\eta_{2}\right\|=L_{V}\left\|\psi_{1}-\psi_{2}\right\|$.

Example 2. Let $(\widetilde{G}, \widetilde{d})$ be an arbitrary nonevolving quasiblock constraining system in $\mathbb{R}^{2}$. Then, $\widetilde{G}$ is a parallelogram. Let $\alpha$ be the obtuse angle in $\widetilde{G}$ and let $\bar{\alpha}=\pi-\alpha$. We can assume without the loss of generality that $(\widetilde{G}, \widetilde{d})$ is generated by vectors $\mathbf{v}_{1}=a \mathbf{e}_{1}$ and $\mathbf{v}_{2}=b\left(\cos \alpha \mathbf{e}_{1}+\sin \alpha \mathbf{e}_{2}\right)$. More specifically, we assume that $\widetilde{G}$ has vertices at $\mathbf{0}, \mathbf{v}_{1}, \mathbf{v}_{2}$, and $\mathbf{v}_{1}+\mathbf{v}_{2}$. Then, $C_{\bar{V}}=\max \{\tan (\alpha / 2), 1\}=\tan (\alpha / 2)=\cot (\bar{\alpha} / 2)$, $K_{\bar{V}}=\sin \alpha=\sin \bar{\alpha}=2 \sin (\bar{\alpha} / 2) \cos (\bar{\alpha} / 2)$, and $\left\|\overline{\mathbf{v}}_{1}+\overline{\mathbf{v}}_{2}\right\|=2$ $\cos (\alpha / 2)=2 \sin (\bar{\alpha} / 2)$ and so $L_{V}=\csc (\bar{\alpha} / 2)$.

Let $\widetilde{\psi}_{1}=-r\left(\cot \alpha \overline{\mathbf{v}}_{1} I_{[0,1)}+\csc \alpha \overline{\mathbf{v}}_{1} I_{[1, \infty)}\right)$ and $\widetilde{\psi}_{2}=-r$ $\left(\csc \alpha \overline{\mathbf{v}}_{2} I_{[0,1)}+\cot \alpha \overline{\mathbf{v}}_{2} I_{[1, \infty)}\right), \quad$ where $\quad r<\min \{-a \tan \alpha, b$ $\cot (\alpha / 2)\}$. Then, using projections as in Example 1, we can evaluate $\widetilde{\phi}_{1}$ and $\widetilde{\phi}_{2}$.

Since $r<-a \tan \alpha$, we have that $\widetilde{\psi}_{1}(0)=-r \cot \alpha \overline{\mathbf{v}}_{1} \in \widetilde{G}$ and so $\left.\widetilde{\phi}_{1}(0)=\pi \sim \widetilde{\psi}_{1}(0)\right)=\widetilde{\psi}_{1}(0)=-r \cot \alpha \overline{\mathbf{v}}_{1}$. On the other hand, $\left.\widetilde{\phi}_{2}(0)=\pi \sim \widetilde{\psi}_{2}(0)\right)=\mathbf{0}$. Therefore, $\tilde{\eta}_{1}(0)$ $=\widetilde{\phi}_{1}(0)-\widetilde{\psi}_{1}(0)=\mathbf{0}$ and $\widetilde{\eta}_{2}^{G}(0)=\widetilde{\phi}_{2}(0)-\widetilde{\psi}_{2}(0)=r \csc \alpha \overline{\mathbf{v}}_{2}$.

Since $\widetilde{\phi}_{1}(0)+\widetilde{\psi}_{1}(1)-\widetilde{\psi}_{1}(0)=\widetilde{\psi}_{1}(1)=-r \csc \alpha \overline{\mathbf{v}}_{1} \notin \widetilde{G}$, $\widetilde{\phi}_{1}(1)=\pi \sim \widetilde{G}_{G}\left(-r \csc \alpha \overline{\mathbf{v}}_{1}\right)=\mathbf{0}$ and $\widetilde{\eta}_{1}(1)=-\widetilde{\psi}_{1}(1)=r \csc \alpha \overline{\mathbf{v}}_{1}$. Since $r<b \cot (\alpha / 2)$, we have that $\widetilde{\phi}_{2}(0)+\widetilde{\psi}_{2}(1)-\widetilde{\psi}_{2}$ $(0)=r\left(\csc \alpha \overline{\mathbf{v}}_{2}-\cot \alpha \overline{\mathbf{v}}_{2}\right)=r \tan (\alpha / 2) \overline{\mathbf{v}}_{2} \in \widetilde{G}, \widetilde{\phi}_{2}(1)=\pi_{\widetilde{G}}$ $\left(r \tan (\alpha / 2) \overline{\mathbf{v}}_{2}\right)=r \tan (\alpha / 2) \overline{\mathbf{v}}_{2}, \quad$ and $\tilde{\eta}_{2}(1)=\widetilde{\phi}_{2}(1)$ $-\widetilde{\psi}_{2}(1)=r \csc \alpha \overline{\mathbf{v}}_{2}$.

Now, $\left\|\widetilde{\eta}_{1}(0)-\widetilde{\eta}_{2}(0)\right\|=\left\|r \csc \alpha \overline{\mathbf{v}}_{2}\right\|=r \csc \alpha$ and $\| \widetilde{\eta}_{1}$ $-\widetilde{\eta}_{2}(1)\|=\| r \csc \alpha \overline{\mathbf{v}}_{1}-r \csc \alpha \overline{\mathbf{v}}_{2}\|=r\|(\tan (\alpha / 2),-1) \|=r \sec$ $(\alpha / 2)=r \csc (\bar{\alpha} / 2)$. Thus, $\left\|\tilde{\eta}_{1}-\tilde{\eta}_{2}\right\|=r \max \{\csc \alpha, \csc$ $(\bar{\alpha} / 2)\}=r \csc (\bar{\alpha} / 2)$. On the other hand, $\left\|\widetilde{\psi}_{1}(0)-\widetilde{\psi}_{2}(0)\right\|=$ $\left\|-r \cot \alpha \overline{\mathbf{v}}_{1}+r \csc \alpha \overline{\mathbf{v}}_{2}\right\|=r\left\|\mathbf{e}_{2}\right\|=r$ and $\left\|\widetilde{\psi}_{1}(1)-\widetilde{\psi}_{2}(1)\right\|=$ $\left\|-r \csc \alpha \overline{\mathbf{v}}_{1}+r \cot \alpha \quad \overline{\mathbf{v}}_{2}\right\|=r\|(-\sin \alpha, \quad \cos \alpha)\|=r$. Therefore, $\left\|\widetilde{\psi}_{1}-\widetilde{\psi}_{2}\right\|=r$ and so $\left\|\widetilde{\eta}_{1}-\widetilde{\eta}_{2}\right\|=\csc (\alpha / 2)\left\|\widetilde{\psi}_{1}-\widetilde{\psi}_{2}\right\|$.

In other words, in this case, $\left\|\widetilde{\eta}_{1}-\widetilde{\eta}_{2}\right\|=L_{V}\left\|\widetilde{\psi}_{1}-\widetilde{\psi}_{2}\right\|$. Because $\widetilde{G}$ represents an arbitrary quasiblock constraining system in $\mathbb{R}^{2}, L_{V}$ is a tight Lipschitz constant in $\mathbb{R}^{2}$.

The next example will demonstrate that the Lipschitz constant in Theorem 2 is not only smaller than the one in Proposition 4 but also exhibits other desirable qualities.

Example 3. We consider a simple example of a quasiblock constraining system in $\mathbb{R}^{2}$, where $\widetilde{G}=\left\{\left(x^{1}+\right.\right.$ $\left.\left.(1 / 2) x^{2},(\sqrt{3} / 2) x^{2}\right): 0 \leq x^{1} \leq 1,0 \leq x^{2} \leq 1\right\}$. In other words, $(\widetilde{G}, \widetilde{d})$ is generated by vectors $\mathbf{v}_{1}=\mathbf{e}_{1}$ and $\mathbf{v}_{2}=(1 / 2) \mathbf{e}_{1}+$ $(\sqrt{3} / 2) \mathbf{e}_{2}$ or by a linear transformation:

$$
T=\left[\begin{array}{cc}
1 & \frac{1}{2} \\
0 & \frac{\sqrt{3}}{2}
\end{array}\right] .
$$

$$
\text { Then, } \angle\left(\overline{\mathbf{v}}_{1}, \overline{\mathbf{v}}_{2}\right)=(\pi / 3), \quad C_{1}^{\lambda}(V)=\tan (\pi / 6)=(\sqrt{3} / 3) \text {, }
$$
$C_{2}^{\lambda}(V)=1$ for any $\lambda$ and so $C_{V}=1$; 
$K_{\bar{V}}=\sin (\pi / 3)=(\sqrt{3} / 2) \quad$ and $\quad\left\|\bar{v}_{1}+\bar{v}_{2}\right\|=\|((3 / 2)$, $(\sqrt{3} / 2)) \|=\sqrt{3}$. Thus, the Lipschitz constant given in (59) is $L_{V}=\sqrt{3} /(\sqrt{3} / 2)=2$.

We can compare it to the Lipschitz constant in (40) of Proposition 4. Since

$$
\begin{aligned}
\|T\| & =\max _{\|\mathbf{x}\| \leq 1}\|T \mathbf{x}\|=\max _{0 \leq \alpha \leq 2 \pi}\|T(\cos \alpha, \sin \alpha)\| \\
& =\max _{0 \leq \alpha \leq 2 \pi}\left\|\left(\cos \alpha+\frac{1}{2} \sin \alpha, \frac{\sqrt{3}}{2} \sin \alpha\right)\right\|,
\end{aligned}
$$

it is enough to maximize the function

$$
g(\alpha)=\left(\cos \alpha+\frac{1}{2} \sin \alpha\right)^{2}+\left(\frac{\sqrt{3}}{2} \sin \alpha\right)^{2}=\sin \alpha \cos \alpha+1
$$

Since $\max \{g(\alpha): 0 \leq \alpha \leq 2 \pi\}=g(\pi / 4)=(3 / 2)$, we get $\|T\|=\sqrt{6} / 2$.

The inverse of $T$ is defined by

$$
T^{-1}\left(x^{1}, x^{2}\right)=\left(x^{1}-\frac{\sqrt{3}}{3} x^{2}, \frac{2 \sqrt{3}}{3} x^{2}\right) \text {. }
$$

A similar analysis to the above shows that

$$
\begin{aligned}
\left\|T^{-1}\right\| & =\max _{0 \leq \alpha \leq 2 \pi}\left\|T^{-1}(\cos \alpha, \sin \alpha)\right\| \\
& =\max _{0 \leq \alpha \leq 2 \pi}\left\|\left(\cos \alpha-\frac{\sqrt{3}}{3} \sin \alpha, \frac{2 \sqrt{3}}{3} \sin \alpha\right)\right\| \\
& =\left\|\left(\cos \frac{2 \pi}{3}-\frac{\sqrt{3}}{3} \sin \frac{2 \pi}{3}, \frac{2 \sqrt{3}}{3} \sin \frac{2 \pi}{3}\right)\right\|=\sqrt{2} .
\end{aligned}
$$

Hence, the Lipschitz constant described in (40) is $\sqrt{n} \cdot\|T\| \cdot\left\|T^{-1}\right\|=\sqrt{6}$.

Thus, for this particular quasiblock constraining system, Theorem 2 provides a better Lipschitz constant than Proposition 4. Moreover, as pointed out in Remark 3, the constant in Proposition 4 depends also on the size of vectors in $V$. In fact, let us examine what happens when $\left\|\mathbf{v}_{1}\right\|$ changes. We consider now a quasiblock constraining system generated by $V=\left\{r \mathbf{e}_{1},(1 / 2) \mathbf{e}_{1}+(\sqrt{3} / 2) \mathbf{e}_{2}\right\}$, where $r>0$. This time

$$
\begin{aligned}
T \mathbf{x} & =T\left(x^{1}, x^{2}\right)=\left(r x^{1}+\frac{1}{2} x^{2}, \frac{\sqrt{3}}{2} x^{2}\right), \\
T^{-1} \mathbf{x} & =T^{-1}\left(x^{1}, x^{2}\right)=\left(\frac{1}{r} x^{1}-\frac{\sqrt{3}}{3 r} x^{2}, \frac{2 \sqrt{3}}{3} x^{2}\right) .
\end{aligned}
$$

Therefore,

$$
\begin{gathered}
\|T\| \geq\|T(1,0)\|=\|(r, 0)\|=r, \\
\left\|T^{-1}\right\| \geq\left\|T^{-1}(0,1)\right\|=\left\|\left(0, \frac{2 \sqrt{3}}{3}\right)\right\|=\frac{2 \sqrt{3}}{3},
\end{gathered}
$$

and so the Lipschitz constant described in (40) of Proposition 4 is $\sqrt{n} \cdot\|T\| \cdot\left\|T^{-1}\right\| \geq \sqrt{2} \cdot r \cdot(2 \sqrt{3} / 3)>r$. In other words, this Lipschitz constant increases without bounds as the magnitude of $\mathbf{v}_{1}$ increases. On the other hand, the Lipschitz constant given in (59) remains unchanged.

\section{Data Availability}

No data were used to support this study.

\section{Conflicts of Interest}

The author declares that there are no conflicts of interest.

\section{References}

[1] A. V. Skorokhod, "Stochastic equations for diffusion processes in a bounded region," Theory of Probability \& Its Applications, vol. 6, no. 3, pp. 264-274, 1961.

[2] H. Tanaka, "Stochastic differential equations with reflecting boundary condition in convex regions," Hiroshima Mathematical Journal, vol. 9, pp. 163-177, 1979.

[3] J. M. Harrison and M. I. Reiman, "Reflected Brownian motion on an orthant," Annals of Probability, vol. 9, pp. 302-308, 1981.

[4] P. L. Lions and A. S. Sznitman, "Stochastic differential equations with reflecting boundary conditions," Communications on Pure and Applied Mathematics, vol. 37, no. 4, pp. 511-537, 1984.

[5] L. Kruk, J. Lehoczky, K. Ramanan, and S. Shreve, "An explicit formula for the Skorokhod map on [0,a]," Annals of Probability, vol. 35, pp. 1740-1768, 2007.

[6] K. Burdzy, W. Kang, and K. Ramanan, "The Skorokhod problem in a time-dependent interval," Stochastic Processes and Their Applications, vol. 119, pp. 428-452, 2009.

[7] M. Slaby, "Explicit representation of the Skorokhod map with time dependent boundaries," Probability and Mathematical Statistics, vol. 30, pp. 29-60, 2010.

[8] M. Slaby, "An explicit representation of the extended Skorokhod map with two time dependent boundaries," Journal of Probability and Statistics, vol. 2010, Article ID 846320, 18 pages, 2010.

[9] M. Slaby, "Explicit Solutions of the extended Skorokhod problems in time-dependednt bounded regions with orthogonal reflection fields," Probability and Mathematical Statistics, vol. 33, no. 1, pp. 121-148, 2013.

[10] H. S. M. Coxeter, Regular Politopes, Dover Publications, Mineola, NY, USA, 1973.

[11] K. Ramanan, "Reflected difusions defined via the extended Skorokhod map," Electronic Journal of Probability, vol. 11, pp. 934-992, 2006.

[12] C. Costantini, E. Gobet, and N. El Karoui, "Boundary sensitivities for diffusion processes in time dependent domains," Applied Mathematics and Optimization, vol. 54, no. 2, pp. 159-187, 2006.

[13] K. Burdzy, Z.-Q. Chen, and J. Sylvester, "The heat equation and reflected Brownian motion in time-dependent domains," Journal of Functional Analysis, vol. 204, no. 1, pp. 1-34, 2003.

[14] L. Slominski and T. Wojciechowski, "Stochastic differential equations with time-dependent reflecting barriers," Stochastics an International Journal of Probability and Stochastic Processes, vol. 85, pp. 27-47, 2013.

[15] K. Nyström and T. Önskog, "The Skorokhod oblique reflection problem in time-dependent domains," Annals of Probability, vol. 38, pp. 2170-2223, 2010. 
[16] W. Kang and K. Ramanan, "On the submartingle problem for reflected diffusionsin domains with piecewise smooth boundaries," Annals of Probability, vol. 45, pp. 404-468, 2017.

[17] D. Lipshutz and K. Ramanan, "On directional derivatives of Skorokhod maps in convex polyhedral domains," Annals of Applied Probability, vol. 28, pp. 688-750, 2018.

[18] K. Ramanan and M. Reiman, "The heavy traffic limit of an unbalanced generalized processor sharing model," Annals of Applied Probability, vol. 18, pp. 22-58, 2008.

[19] Y. Saisho, "Stochastic differential equations for multi-dimensional domain with reflecting boundary," Probability Theory and Related Fields, vol. 74, no. 3, pp. 455-477, 1987.

[20] P. Dupuis and H. Ishii, "On lipschitz continuity of the solution mapping to the skorokhod problem, with applications," Stochastics and Stochastic Reports, vol. 35, no. 1, pp. 31-62, 1991.

[21] P. Dupuis and K. Ramanan, "Convex duality and the skorokhod problem. I," Probability Theory and Related Fields, vol. 115, no. 2, pp. 153-195, 1999.

[22] P. Dupuis and K. Ramanan, "Convex duality and the skorokhod problem. II," Probability Theory and Related Fields, vol. 115, no. 2, pp. 197-236, 1999.

[23] C. Costantini, "The Skorohod oblique reflection problem in domains with corners and application to stochastic differential equations," Probability Theory and Related Fields, vol. 91, no. 1, pp. 43-70, 1992. 